\title{
Structural basis of sialyltransferase activity in trypanosomal sialidases
}

\author{
Alejandro Buschiazzo ${ }^{1,2}$, Gisele A.Tavares ${ }^{1,3}$, \\ Oscar Campetella², Silvia Spinelli ${ }^{4}$, \\ Marfa L.Cremona ${ }^{2}$, Gastón París ${ }^{2}$, \\ Maria Fernanda Amaya ${ }^{1}$, Alberto C.C.Frasch ${ }^{2,5}$ \\ and Pedro M.Alzari ${ }^{1,5}$ \\ ${ }^{1}$ Unité de Biochimie Structurale, Institut Pasteur, 25 rue du Dr Roux, \\ 75724 Paris, ${ }^{4}$ AFMB-CNRS UPR 9039, 31 Chemin J. Aiguier, \\ 13402 Marseille, France and ${ }^{2}$ Instituto de Investigaciones \\ Biotecnológicas-Universidad Nacional de San Martín, CC30, \\ 1650 San Martín, Argentina \\ ${ }^{3}$ Present address: Department of Molecular Biophysics and \\ Biochemistry, Yale University, 260 Whitney Avenue JWG423, \\ New Haven, CT 06511, USA \\ ${ }^{5}$ Corresponding authors \\ e-mail: alzari@pasteur.fr or cfrasch@iib.unsam.edu.ar
}

The intracellular parasite Trypanosoma cruzi, the etiological agent of Chagas disease, sheds a developmentally regulated surface trans-sialidase, which is involved in key aspects of parasite-host cell interactions. Although it shares a common active site architecture with bacterial neuraminidases, the T.cruzi enzyme behaves as a highly efficient sialyltransferase. Here we report the crystal structure of the closely related Trypanosoma rangeli sialidase and its complex with inhibitor. The enzyme folds into two distinct domains: a catalytic $\beta$-propeller fold tightly associated with a lectin-like domain. Comparison with the modeled structure of T.cruzi trans-sialidase and mutagenesis experiments allowed the identification of amino acid substitutions within the active site cleft that modulate sialyltransferase activity and suggest the presence of a distinct binding site for the acceptor carbohydrate. The structures of the Trypanosoma enzymes illustrate how a glycosidase scaffold can achieve efficient glycosyltransferase activity and provide a framework for structure-based drug design.

Keywords: inhibitor complex/sialidase/sialyltransferase/ Trypanosoma cruzi/X-ray structure

\section{Introduction}

The trans-sialidase from Trypanosoma cruzi (TcTS), the protozoan agent of Chagas disease in the American continent, is a member of a family of glycoproteins expressed on the parasite's surface and shed into the medium (Frasch, 1994; Schenkman et al., 1994), thus constituting one of few natural examples of surface glycosyltransferases in eukaryotes. Trypanosomes are unable to synthesize sialic acid and use this enzyme to scavenge the monosaccharide from host glycoconjugates to sialylate mucin-like acceptor molecules present in the parasite plasma membrane (Previato et al., 1985). Sialic acid in mucins has been implicated in key aspects of parasite-host cell interactions such as cell adhesion and invasion (Schenkman et al., 1991) and resistance to non-specific complement attack (Tomlinson and Raper, 1998). Given the involvement of trans-sialidase in T.cruzi infection of humans and the absence of a similar enzyme in eukaryotic cells, TcTS constitutes a good target for the development of compounds useful in controlling the infection.

The TcTS protein family is encoded by $\sim 140$ genes in the Trypanosoma genome (Cremona et al., 1999), but many of these code for inactive protein products (Schenkman et al., 1994; Cremona et al., 1995). The protein consists of a $70 \mathrm{kDa}$ globular core conveying the enzymatic activity followed by a variable number of immunogenic repeats (SAPA; Parodi et al., 1992). TcTS has been classified as an exo- $\alpha$-sialidase (EC 3.2.1.18) (Roggentin et al., 1993; Vimr, 1994) and, according to its primary structure (Campetella et al., 1994), it belongs to glycosidase family 33 (Henrissat and Bairoch, 1993; Henrissat, 1998), which also includes several bacterial sialidases. However, TcTS behaves as a sialyltransferase in vivo and in vitro in the presence of suitable sugar acceptor substrates (terminal $\beta$-galactose in glycoconjugates) (Parodi et al., 1992; Vandekerckhove et al., 1992; Ferrero-García et al., 1993; Schenkman et al., 1994). As in other glycosidase-catalyzed glycoside synthesis reactions, the transfer reaction catalyzed by trans-sialidase (Figure 1) is different from that of typical sialyltransferases (EC 2.4.99.X), which act with inversion of configuration and require sugar-nucleotides (i.e. CMP- $N$-acetylneuraminate) as the monosaccharide donor (Paulson and Colley, 1989).

The reasons why trans-sialidase is more efficient in transferring than in hydrolyzing terminal sialic acids are unknown. The primary structure of the 640 amino acids of TcTS on the N-terminal region (excluding the C-terminal SAPA repeats) predicts the presence of a catalytic domain of 380 amino acids that is $30 \%$ identical to some bacterial sialidases (Hoyer et al., 1992; Cremona et al., 1995). Most of the amino acids present in the active site of microbial sialidases are conserved within this TcTS domain (Pereira et al., 1991; Pollevick et al., 1991; Uemura et al., 1992; Cremona et al., 1995). The remaining 260 residues that follow this catalytic domain constitute a region of unknown structure/function that has no detectable homology with known protein sequences.

A closely related American trypanosome parasite, Trypanosoma rangeli (D'Alessandro-Bacigalupo and Gore Saravia, 1992), expresses a surface sialidase (TrSA) that is, like TcTS, the product of a multi-gene family encoding both active and inactive protein products (Buschiazzo et al., 1993, 1997; Smith et al., 1996). Although TrSA is very similar in amino acid sequence to the globular core of TcTS ( 70\% identity for 640 amino 


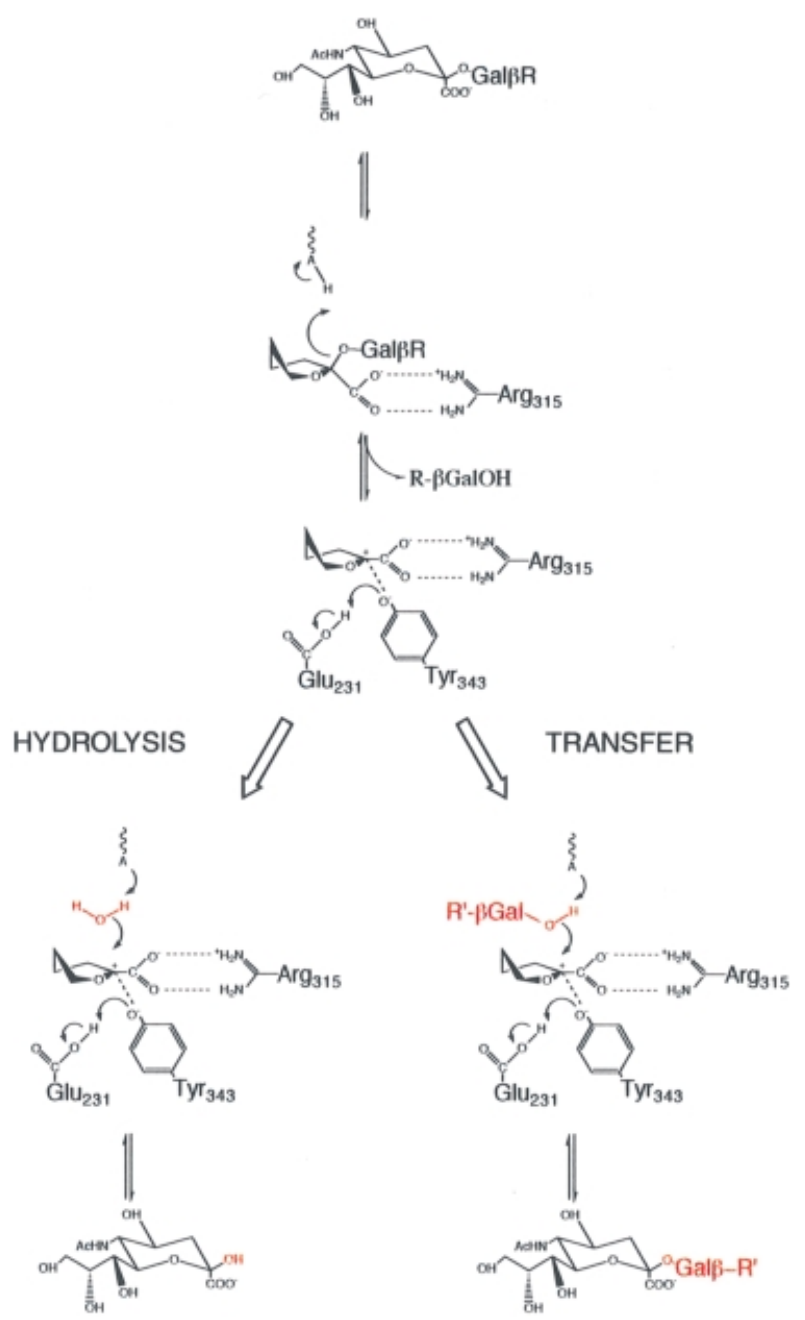

Fig. 1. Scheme showing the hydrolysis and transfer reactions catalyzed by TcTS, based on the mechanism proposed for influenza virus sialidase (Chong et al., 1992; Burmeister et al., 1993). R and R' stand for any molecule bound to the terminal $\beta$-galactose. The proton donor or general acid has been tentatively assigned to Asp60 (Chong et al., 1992), a strictly conserved residue in sialidases that is favorably positioned to fulfill this role. Key residues involved in sialic acid binding or catalysis are depicted. The second part of the reaction proceeds along one of two alternative pathways depending on the nature of the nucleophile: a water molecule or a second glycoconjugate $\mathrm{R}^{\prime}-\beta \mathrm{Gal}$ (both colored in red). The first pathway results in glycosyl hydrolysis with production of free $\alpha$-sialic acid. In the second (and most favored) case, the overall outcome of the reaction is the transfer of sialic acid from the donor substrate R- $\beta \mathrm{Gal}-$ $\alpha-2,3-$ Sial to the acceptor glycoconjugate $\mathrm{R}^{\prime}-\beta \mathrm{Gal}$.

acids), it lacks a repetitive $\mathrm{C}$-terminal domain and is completely devoid of trans-sialidase activity (Pontes-deCarvalho et al., 1993b). Comparative sequence analysis shows that the amino acid differences between $\operatorname{TrSA}$ and TcTS are distributed along the entire sequence, thus providing no further clues about the regions of the primary structure that modulate transferase activity.

Given their high sequence similarity and their different enzymatic activities, TrSA and TcTS constitute a unique model to study the molecular basis of this sugar-nucleotideindependent trans-sialylation activity. We describe here the crystal structure of $\operatorname{TrSA}$ in its free and inhibitorcomplexed forms, and compare the structure with the homology model of TcTS and other sialidases. The threedimensional structures and mutagenesis experiments
Table I. Crystallographic data and refinement statistics for unliganded TrSA and its complex with the inhibitor 2-deoxy-2,3-didehydro- $N$ acetylneuraminic acid (DANA)

\begin{tabular}{lll}
\hline & TrSA & TrSA-DANA \\
\hline Data collection & & \\
Synchrotron beamline & BW7 & D41 LURE \\
& EMBL-DESY & \\
Wavelength & $0.95 \AA$ & $1.375 \AA$ \\
No. of measured reflections & 210923 & 112960 \\
No. of unique reflections & 38612 & 17063 \\
Resolution & $20-2.2 \AA$ & $15-2.9 \AA$ \\
& $(2.24-2 \AA)$ & $(2.98-2.9 \AA)$ \\
Completeness & $99.3 \%(97.7 \%)$ & $99.9 \%(99.8 \%)$ \\
$R$-merge & $12.6 \%(35.4 \%)$ & $13.8 \%(30.6 \%)$ \\
Percentage of references & $66.8 \%(43.3 \%)$ & $82.7 \%(69.4 \%)$ \\
with I $>$ 2 $\sigma(\mathrm{I})$ & & \\
Refinement & & \\
Resolution range & $10-2.2 \AA$ & $8-2.9 \AA$ \\
No. of reflections & 38207 & 16275 \\
$R$-factor & $19.4 \%$ & $20.0 \%$ \\
Free $R$-factor & $26.3 \%$ & $31.4 \%$ \\
R.m.s. (bond lengths) & $0.018 \AA$ & $0.019 \AA$ \\
R.m.s. (bond angles) & $0.045 \AA$ & $0.063 \AA$ \\
Protein atoms & 4769 & 4759 \\
Sugar atoms & 70 & 90 \\
Water molecules & 365 & 40 \\
\hline
\end{tabular}

suggest the presence of a distinct binding site for the acceptor saccharide in trans-sialidase.

\section{Results}

The overall structure of T.rangeli sialidase

The crystal structure of $\operatorname{TrSA}$ has been determined at $2.2 \AA$ resolution, and its complex with the inhibitor 2,3-didehydro-2-deoxy- $N$-acetylneuraminic acid (DANA) at $2.9 \AA$ resolution (Table I). The globular core of TrSA folds into two distinct structural domains (Figure 2). The N-terminal catalytic domain (residues 1-372) displays a canonical ' $\beta$-propeller' topology (Varghese et al., 1983; Vimr, 1994) similar to that of bacterial and viral sialidases (Table II). It is connected by a long $\alpha$-helical segment (residues 373-395) to the second C-terminal domain (residues 398-614), which shows the characteristic $\beta$-barrel topology of plant lectins (Table II). The protein ends with a second $\alpha$-helix, which in TcTS is followed by the immunogenic SAPA repeats.

The catalytic and lectin-like domains of TrSA are tightly associated with each other. The association buries $2620 \AA^{2}$ of exposed molecular surface: $1290 \AA^{2}$ from the catalytic domain (including the connecting $\alpha$-helix) and $1330 \AA^{2}$ from the lectin-like domain. This extended contact surface area is substantially greater than that observed for other microbial sialidases that also have a lectin-like domain flanking the catalytic domain, such as the Vibrio cholerae and Macrobdella decora enzymes (Crennell et al., 1994; Luo et al., 1998), for which the interaction between the two domains buries a total surface area of 1300-1600 $\AA^{2}$.

The electron density maps of TrSA show sugar residues attached to all five predicted N-glycosylation sites (at asparagine residues 15, 24 and 115 in the catalytic domain, and 429 and 614 in the lectin-like domain). In each case, the innermost monosaccharide is visible in density and was modeled as $N$-acetylglucosamine (GlcNAc) (Figure 2). 

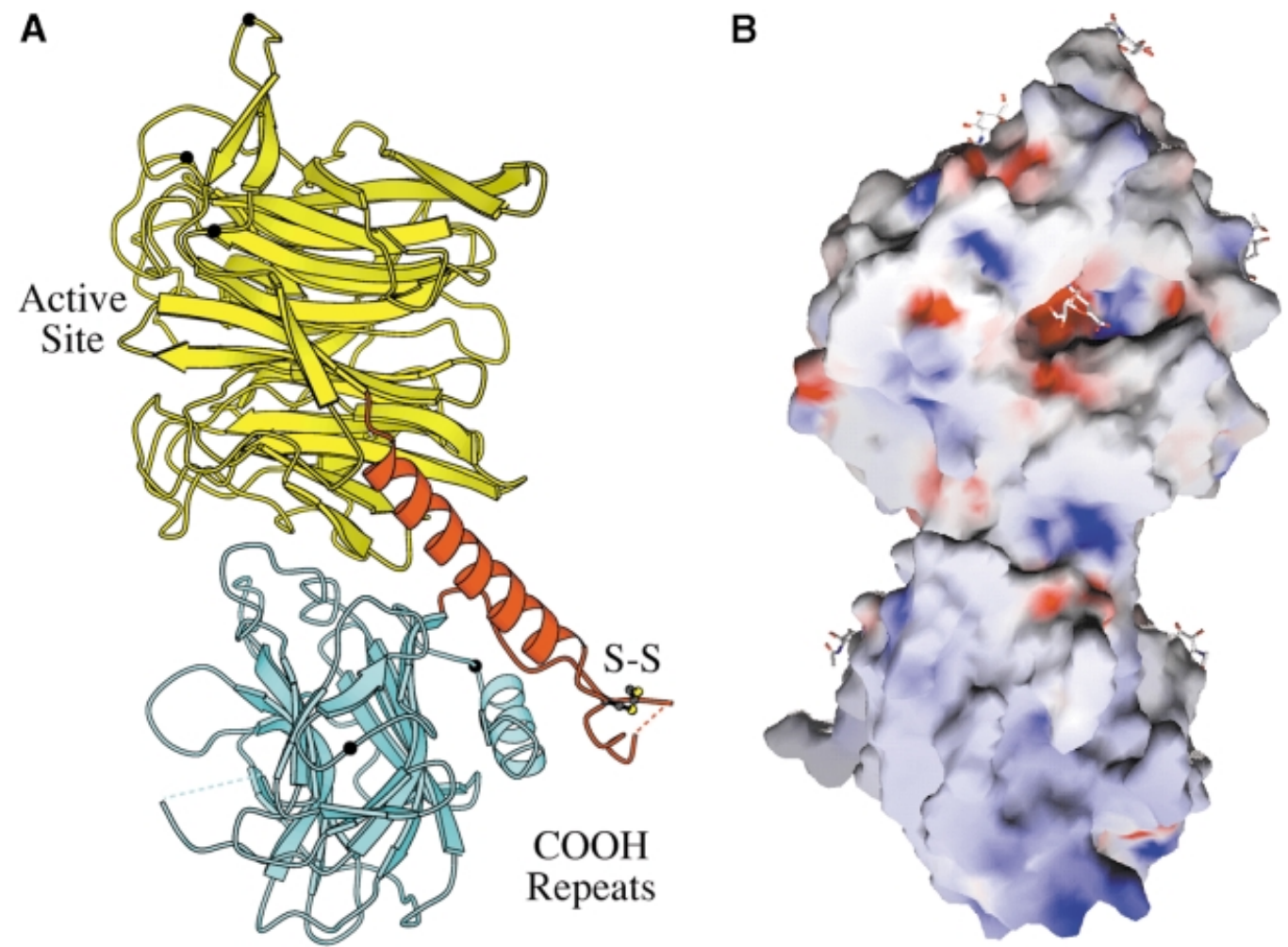

Fig. 2. Overall structure of TrSA. The structure consists mainly of $\beta$-sheets (43 strands), with only two $\alpha$-helices, one connecting the two domains and the other at the C-terminus. (A) Ribbon model showing the neuraminidase fold (yellow), the connecting $\alpha$-helix (red) and the lectin-like domain (cyan). In TcTS, the C-terminal helix is followed by the SAPA repeats. One disulfide bridge (Cys397-Cys411) is found at the junction between the two structural domains (yellow spheres) and is conserved in TcTS. The five N-glycosylation sites are represented by black spheres. (B) Molecular surface colored by electrostatic potential, with the innermost GlcNAc residues and DANA (at the center) shown as stick figures. This view is rotated $90^{\circ}$ about the vertical axis relative to the view shown in (A).

Table II. R.m.s. deviations of the catalytic and lectin-like domains of TrSA when compared with other proteins. The PDB codes and the number of topologically equivalent residues giving rise to the r.m.s. deviations are given in parentheses

\begin{tabular}{|c|c|c|}
\hline & R.m.s. & $\begin{array}{l}\text { Sequence } \\
\text { identity }\end{array}$ \\
\hline \multicolumn{3}{|l|}{ Catalytic domain (residues $1-372$ ) } \\
\hline $\begin{array}{l}\text { Salmonella typhimurium } \\
\text { sialidase (2SIM) }\end{array}$ & $1.48 \AA(314)$ & $29 \%$ \\
\hline Vibrio cholerae sialidase (1KIT) & $1.60 \AA(275)$ & $21 \%$ \\
\hline $\begin{array}{l}\text { Micromonospora viridifaciens } \\
\text { sialidase (1EUR) }\end{array}$ & $1.62 \AA(278)$ & $16 \%$ \\
\hline $\begin{array}{l}\text { Macrobdella decora (leech) } \\
\text { sialidase (1SLL) }\end{array}$ & $1.70 \AA(281)$ & $19 \%$ \\
\hline Influenza virus sialidase (1IVF) & $2.11 \AA ̊(178)$ & $11 \%$ \\
\hline \multicolumn{3}{|l|}{ Lectin-like domain (residues 398-614) } \\
\hline Bacillus $\beta$-glucanase (2AYH) & $2.7 \AA(153)$ & $8 \%$ \\
\hline Tetanus neurotoxin (1A8D) & $2.9 \AA(166)$ & $8 \%$ \\
\hline V.cholerae sialidase (1KIT) & $2.9 \AA(160)$ & $8 \%$ \\
\hline Serum amyloid $\mathrm{P}$ component & $2.9 \AA ̊(144)$ & $10 \%$ \\
\hline M.decora sialidase (1SLL) & $3.0 \AA ̊(143)$ & $14 \%$ \\
\hline Griffonia simplicifolia lectin (1LED) & $3.3 \AA(158)$ & $9 \%$ \\
\hline Bovine S-lectin ${ }^{\mathrm{b}}$ (1SLT) & $2.6 \AA ̊$ (117) & $12 \%$ \\
\hline Human galectin- $3^{\mathrm{b}}(1 \mathrm{~A} 3 \mathrm{~K})$ & $2.7 \AA(120)$ & $14 \%$ \\
\hline
\end{tabular}

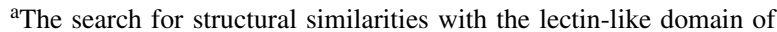
TrSA was carried out using DALI (Holm and Sander, 1994).

${ }^{\mathrm{b}}$ The $\mathrm{N}$-terminal $\beta$-strand is missing in the animal lectins $(1 \mathrm{~A} 3 \mathrm{~K}$, 1SLT).
All sugar chains were located far from the active site cleft and play no role in catalysis since recombinant, nonglycosylated TrSA and TcTS retain their enzymatic activities (Campetella et al., 1994; Buschiazzo et al., 1997).

A conserved motif SXDXGXTW, known as the 'Asp box' and common to all non-viral sialidases (Taylor, 1996), occurs in the loops connecting the third and fourth strands of $\beta$-sheets 1,3 and 4 of the catalytic domain (positions 71-78, 211-218 and 257-264). As observed in bacterial sialidases, these motifs have a well-conserved structure: all main-chain atoms can be superimposed between different loops with r.m.s. fit deviations of 0.28-0.33 ̊. A fourth degenerate Asp box (Pereira et al., 1991 ) lies in a $\beta$-hairpin of the lectin-like domain (residues 518-525), but its overall structure is much less conserved (the r.m.s. fit deviations of main-chain atoms from the three other Asp boxes is $>2.5 \AA$ ).

\section{The active site}

The structure of $\mathrm{TrSA}$ in complex with the inhibitor DANA (Figure 3) revealed that critical active site residues conserved in other microbial sialidases (Crennell et al., 1993; Taylor, 1996) display a similar structural arrangement in the N-terminal catalytic domain of trypanosome enzymes. These invariant residues include an arginine triad (Arg36, Arg246 and Arg315) that binds to the carboxylate group common to all sialic acid derivatives, a glutamic acid (Glu358) that stabilizes one of the arginine 

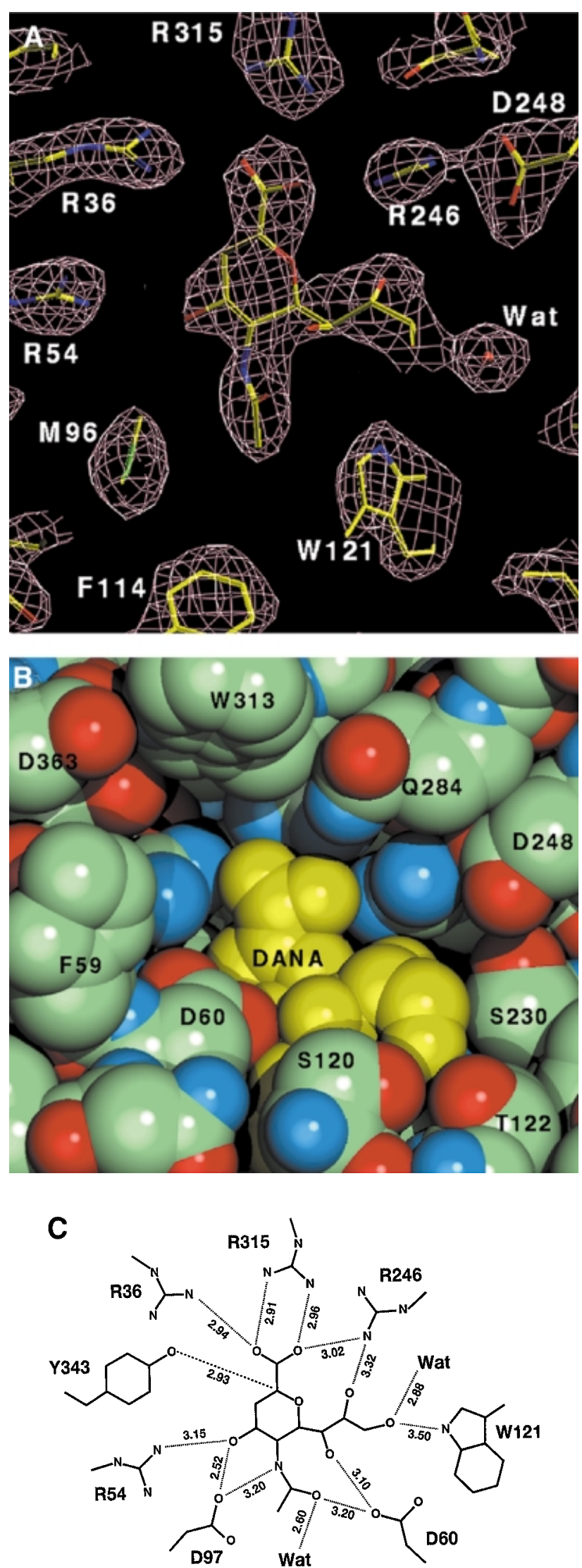

Fig. 3. Structure of TrSA in complex with DANA. (A) Final electron density map at $2.9 \AA$ resolution (contoured at $1.5 \sigma$ ). (B) Top view of the active site pocket with the bound inhibitor (in yellow). (C) Scheme showing enzyme-inhibitor hydrogen bonding interactions.

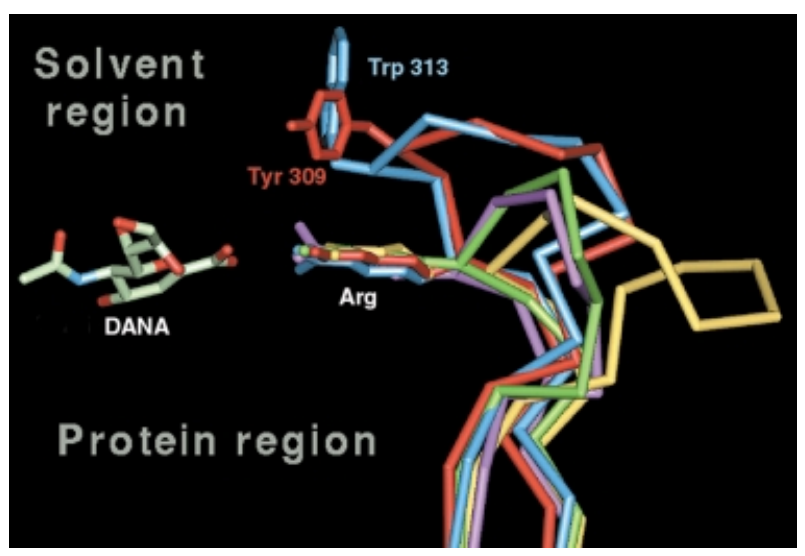

Fig. 4. Superposition of the loop connecting strands 3-4 of the fifth $\beta$-sheet in the catalytic domain of microbial sialidases. In enzymes specific for sialyl- $\alpha(2,3)$-linked oligosaccharides such as T.rangeli (shown in blue) and S.typhimurium (red) sialidases, the loop extends over the conserved triad of arginine residues and includes an aromatic residue (Trp or Tyr) that could interact with substrate. This extension is missing in enzymes with dual $\alpha(2,3)-\alpha(2,6)$ specificity, such as V.cholerae (yellow), Micromonospora viridifaciens (green) and influenza virus (magenta) sialidases. Also shown in the figure is one of the three arginine residues forming salt bridge interactions with the carboxylate group of DANA.

side chains, a negatively charged group (Asp60), which approaches the bound sialic acid from the solvent side and has been proposed as a possible proton donor in the hydrolytic reaction, and two essential residues at the bottom of the site (Glu231, Tyr343), which are well positioned to stabilize a putative sialosyl cation intermediate. The hydroxyl group of Tyr343 is in contact with the $\mathrm{C} 2$ carbon of the inhibitor sugar ring in the structure of the TrSA-DANA complex.

Additional TrSA residues that interact with the inhibitor include Met96, Phe114, Trp121 and Val177, all of which define a hydrophobic pocket that accommodates the $\mathrm{N}$ acetyl group of sialic acid, and two charged residues (Arg54, Asp97), which are involved in hydrogen bonding interactions with the hydroxyl group $\mathrm{OH}-4$ of DANA (Figure 3C). Towards the outer region of the active site cleft, the exposed aromatic side chain of Trp313 is well positioned to make stacking interactions with the leaving group of the substrate (Figure 3B), as suggested by recent mutagenesis results (Chuenkova et al., 1999). Unfavorable interactions of this Trp residue with sialyl- $\alpha(2,6)$-linked oligosaccharides could account for the higher specificity of TrSA (and TcTS) towards sialyl- $\alpha(2,3)$ substrate analogs. The aromatic side chain is part of a lip, which extends over the arginine triad in trypanosomal and Salmonella sialidases (Crennell et al., 1996), but that is absent (due to a shorter loop) in sialidases with dual $\alpha(2,3)-\alpha(2,6)$ specificity (Figure 4$)$.

\section{Comparison of trypanosome sialidase and trans- sialidase}

The protein sequences of the TrSA and TcTS globular cores are $70 \%$ identical. Over two-thirds of the 188 amino acid differences correspond to residues totally or partially exposed to the solvent, which represent $\sim 40 \%$ of the total molecular surface and can therefore account for the lack of cross-reactivity between specific neutralizing antibodies (Buschiazzo et al., 1997). However, the highly conservat- 

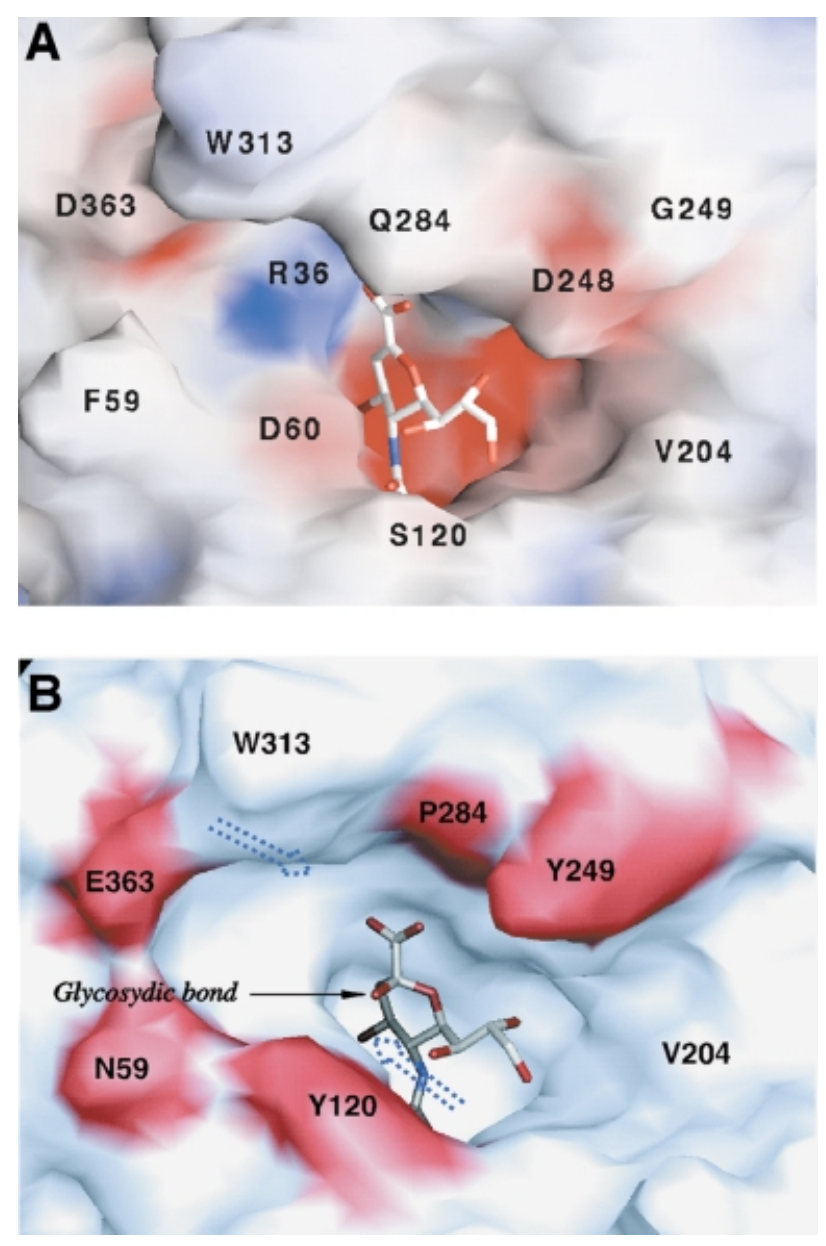

Fig. 5. The active site cleft of trypanosomal sialidases. (A) Structure of the TrSA-inhibitor complex colored according to charge. (B) Model of TcTS with bound sialic acid. Amino acid differences between TrSA and TcTS at the molecular surface (colored in red) involve potential substrate-contacting residues Ser120-Tyr, Gln284-Pro, Gly249-Tyr, Asp363-Glu and Phe59-Asn. Putative binding sites for the sialic acid donor and acceptor substrates, respectively, close to the aromatic side chains of Trp313 and Tyr120 are indicated by dashed arrows (see the text for details).

ive character of internal substitutions, the essentially identical interface between the catalytic and the lectinlike domains, and the lack of insertions or deletions between TrSA and TcTS indicate that the two enzymes share a very similar three-dimensional structure.

Comparison of the crystal structure of $\operatorname{TrSA}$ with the modeled structure of TcTS emphasizes the crucial role of a few amino acid residues within the substrate-binding cleft in modulating the sialyltransferase activity, since the organization of domains in both trypanosomal enzymes (Figure 2) precludes a direct role of the lectin-like domain in the catalytic activity. The center of the inhibitor-binding site (including all sialidase-conserved amino acid residues) is similar in TrSA and TcTS. However, critical substitutions are observed at the periphery of the cleft (Figure 5). The inhibitor-contacting residue Ser120 in TrSA is substituted by tyrosine in TcTS, thus introducing a bulkier, aromatic side chain in close proximity to the substrate. Furthermore, two additional amino acid substitutions at the surface of the cleft, Gln284-Pro and Gly249-Tyr, generate a shallow depression in TcTS, immediately above

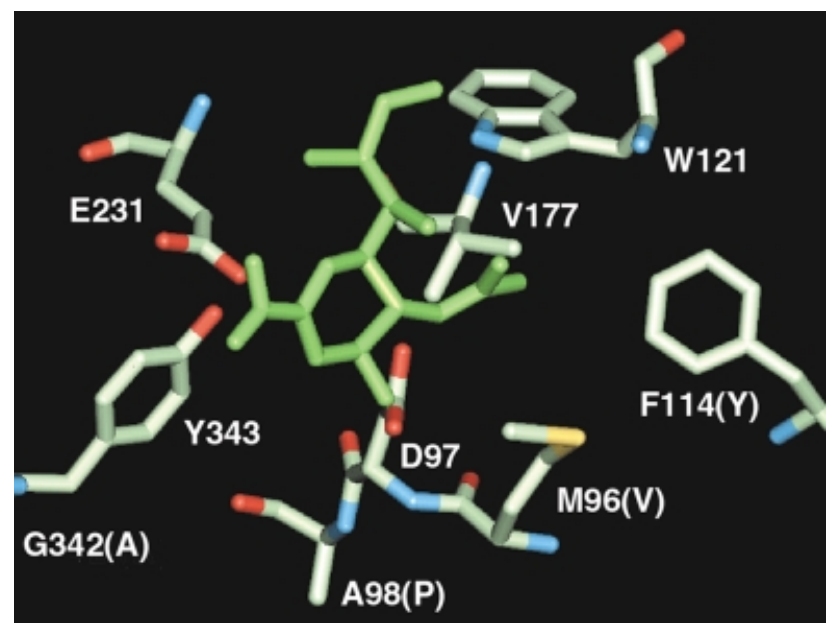

Fig. 6. View of the $\operatorname{TrSA}$ structure showing internal amino acid positions close to the bound inhibitor (shown in green) that are mutated in TcTS. These amino acid substitutions between trypanosomal sialidase and trans-sialidase (TcTS residues are indicated in parentheses) impose different constraints on sialic acid binding to the active site cleft.

the carboxylate group of sialic acid (Figure 5B). These three amino acid substitutions certainly affect proteincarbohydrate interactions and might provide an alternative binding site for the acceptor saccharide, different from that occupied by the leaving aglycon (see below).

Three of the four amino acid residues that define the hydrophobic pocket occupied by the $N$-acetyl group of the inhibitor are also different in TcTS (Figure 6): Val96 (Met in TrSA), Tyr114 (Phe) and Leu177 (Val). In particular, the smaller side chain at position 96 of TcTS could allow a conformational rearrangement of Arg94 (whose side chain is completely buried in $\operatorname{TrSA}$ ), bringing its $\delta$-guanido group towards the surface of the cleft, close to the carboxylic acid groups of Asp52 and Asp97. Other internal substitutions close to the reaction center may also influence the enzymatic activity. At the bottom of the substrate-binding cleft, three conserved polar side chains (Tyr343, Glu231 and Asp97) are directly involved in substrate binding and catalysis. The substitutions Met96Val and Ile37-Leu, and the constraints imposed by a proline residue at position 98 of TcTS (replacing alanine), could sterically affect the position and conformation of Asp97 (see Figure 6). On the other hand, the substitution Gly342-Ala might affect the conformation of the adjacent Tyr343 since Gly342 displays main-chain dihedral angles in the $\operatorname{TrSA}$ structure $\left(\phi=143^{\circ}, \psi=-131^{\circ}\right)$, which are energetically unfavorable for bulkier residues.

On the opposite side to the reaction center, a wide cleft filled with solvent extends deep within the TrSA structure. This cleft is connected through a network of hydrogenbonded water molecules to the base of catalytic residues Tyr343 and Glu231 (Figure 7), forming a water channel that runs through the center of the catalytic domain. This channel appears to be absent in other microbial sialidases due to tighter packing of the $\beta$-sheets. The substitutions Val180-Ala and Thr39-Ala (and probably also Ile37-Leu) create internal cavities that tend to enlarge the volume of the channel in TcTS and could therefore allow water molecules from the bulk solvent to reach the active site. 


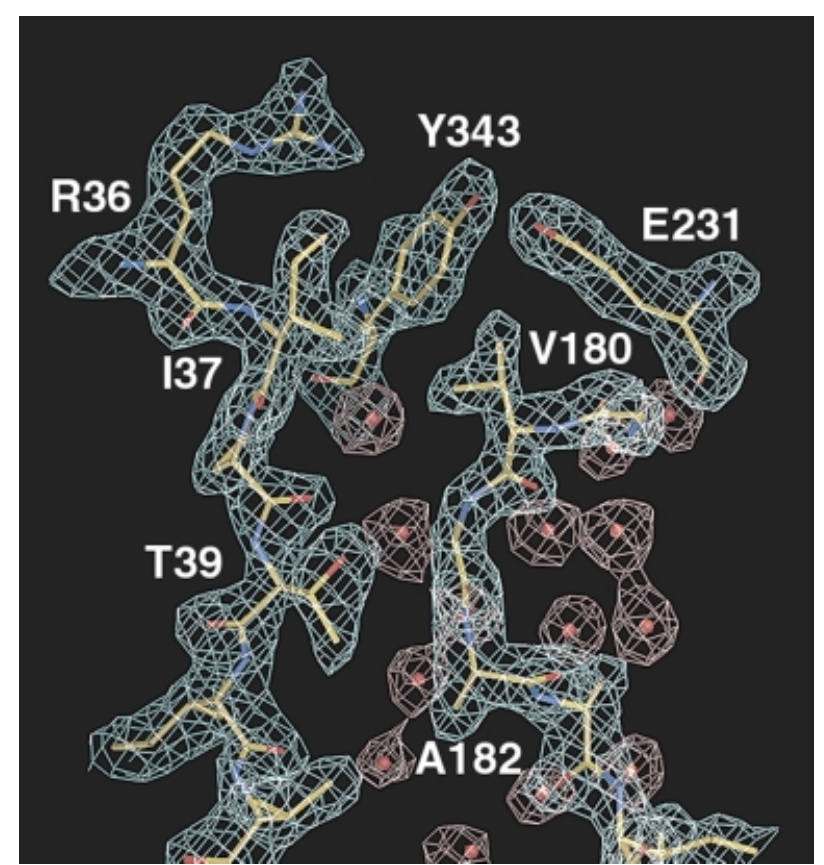

Fig. 7. View of the final $\left(2 F_{\mathrm{o}}-F_{\mathrm{c}}\right)$ electron density map of $\operatorname{TrSA}$ (contoured at $1.5 \mathrm{~s}$ ) showing two central $\beta$-strands of the sialidase domain. A network of hydrogen-bonded water molecules (shown in red) connects the two catalytic residues Tyr343 and Glu231 (top) with a wide cavity filled with solvent at the opposite side of the molecule (bottom). Note that the substitution of TrSA residues Thr39 and Val180 (immediately below the active site cleft) by alanine residues would increase the internal volume accessible to water molecules in TcTs.

\section{Mutagenesis}

To test the hypotheses derived from the structural models, point mutants were generated by exchanging residues among TcTS and TrSA, and the mutated recombinant proteins were assayed for sialidase and trans-sialidase activity (Table III). Exchange of Tyr120 by a serine residue in TcTS significantly decreases the wild-type trans-sialidase/sialidase activity ratio, whereas the inverse mutation in TrSA (Ser120-Tyr) does not produce a significant effect on the hydrolysis reaction. Given the favorable position of the aromatic side chain of Tyr120 to interact with substrate (Figure 5B), the mutagenesis results suggest that this tyrosine residue could define a distinct binding site specific for the terminal $\beta$-galactose acceptor, different from that (towards Trp313) occupied by the substrate donor. A sugar molecule making stacking interactions with Tyr120 would approach the anomeric carbon of sialic acid from its $\alpha$-face, in agreement with the retaining (double displacement) mechanism common to all microbial sialidases.

All other mutants led to opposite effects in TcTS and TrSA (Table III). The single mutant Pro284-Gln and the double mutant Tyr249-Gly/Pro284-Gln significantly affected both hydrolase and transferase activities of TcTS, rendering it inactive, while the Gly249-Tyr/Gln284-Pro double mutation in TrSA significantly increased sialidase activity. The inverse situation was observed for the internal substitutions at positions 39 and 180 within the putative water channel: exchange of the two alanine residues at these positions in TcTS by Thr39 and Val180 retained
$20 \%$ sialidase and $100 \%$ trans-sialidase activities, whereas the inverse substitutions in $\operatorname{TrSA}$ abolished the enzymatic activity. These opposite effects could be the result of different stereochemical constraints upon binding of the sialic acid moiety, in agreement with the observed substitutions close to the catalytic residues (Figures 5B and 6) and the much lower DANA inhibition of TcTS (Vandekerckhove et al., 1992).

\section{Discussion}

The structures of TrSA and TcTS share a common organization in two domains: an N-terminal catalytic domain and a C-terminal lectin-like domain. The latter includes what has previously been identified as a fibronectin type III-like (Fn3) region (Pereira et al., 1991). Previous results have suggested that an intact Fn3-like domain would be required for expression of enzymatically active products from TcTS (Schenkman et al., 1994) and TrSA (Smith et al., 1996). It has also been suggested that the C-terminal region could contain the binding site for the galactosecontaining acceptor molecule (Ribeirao et al., 1997), and that a conserved sequence motif (VTVxNVxLYNR) within this region could be close to the active site cleft in the three-dimensional structure of TcTS (Smith and Eichinger, 1997). However, the structure obtained in this work indicates that the C-terminal region of trypanosomal enzymes folds into a lectin-like domain that is not directly involved in the reaction mechanism. Moreover, similar lectin-like domains were also found in V.cholerae and M.decora sialidases (Table II), but the modes of association with the catalytic domain are different for each enzyme, also arguing against the involvement of the lectin-like domain in catalysis. Instead, the lectin-like domain may be involved in carbohydrate recognition, since the concave face of the curved $\beta$-sheet in TrSA (where most structurally equivalent lectin domains have their sugar-binding sites) was found to be accessible to the solvent. Although further work is necessary to exclude other possibilities, the need for an intact lectin-like domain to have full enzymatic activity is probably related to the extended contact surface between the two structural domains, as discussed above.

The overall pattern of protein-inhibitor interactions in the structure of the TrSA-DANA complex (Figure 3C) is very similar to that observed for Salmonella typhimurium sialidase in complex with the same inhibitor (Crennell et al., 1993). This similarity contrasts with the strikingly different kinetic reaction rates of these two enzymes (Crennell et al., 1993; Buschiazzo et al., 1997), and argues against a previous hypothesis that accounted for the different turnover rates between bacterial and viral neuraminidases on the basis of different interactions with the glycerol moiety of bound sialic acid (Crennell et al., 1993). Indeed, the comparison of trypanosomal and viral enzymes (which show comparable, low turnover rates in spite of dissimilar interactions with DANA) suggests that subtle rearrangements within the sialic acid-binding cleft and/or different interactions with the departing aglycon may significantly influence the reaction rates of hydrolysis.

The reasons why trans-sialidase acts so efficiently as a transferase have been summarized in two alternative models (Schenkman et al., 1994; Ribeirao et al., 1997; Smith and Eichinger, 1997), both consistent with the 
Table III. Sialidase (SA) and trans-sialidase (TS) activities of mutant recombinant proteins

\begin{tabular}{lccl}
\hline Enzyme & SA activity & TS activity & TS/SA ratio \\
\hline TcTS wild type & $96 \pm 4(100)$ & $730 \pm 11(100)$ & 7.6 \\
TcTS Tyr120-Ser & $12.3 \pm 0.5(12.8)$ & $4.3 \pm 0.8(0.6)$ & 0.35 \\
TcTS Pro284-Gln & 0 & 0 & - \\
TcTS Tyr249-Gly, Pro284-Gln & 0 & 0 & - \\
TcTS Ala39-Thr, Ala180-Val & $85 \pm 5(88.6)$ & $793 \pm 47(109)$ & 0 \\
TrSA wild type & $1880 \pm 110(100)$ & 0 & 0 \\
TrSA Ser120-Tyr & $956 \pm 12(51)$ & 0 & 0 \\
TrSA Gly249-Tyr, Gln284-Pro & $16970 \pm 880(902)$ & 0 & 0 \\
TrSA Thr39Ala, Val180-Ala & 0 & 0 & \\
\hline
\end{tabular}

Activities are expressed as nmol sialic acid/min/mg (free sialic acid for SA; amount of sialic acid transferred to lactose as acceptor for TS). The percentage of activity referred to wild-type controls is indicated in parentheses. In the case of the TcTS Tyr120-Ser mutant, the enzymatic activities were further assayed at different concentrations of substrates. At $V_{\max }$ the percentage of TS activity with respect to wild-type TcTS was $0.6 \%$, whilst the SA activity ratio was $45 \%$

reaction mechanism shown in Figure 1. (i) Trans-sialidase might form a more stable sialosyl-enzyme complex than a typical sialidase. The enzyme would then retain the sialyl residue for a longer time, sufficient to release the aglycon moiety and bind the acceptor sugar with a terminal $\beta$-galactose residue. This scenario is plausible since all retaining glycosidases can also function as transglycosidases (Koshland, 1953). (ii) Trans-sialidase may have acquired a new, distinct binding site for the acceptor $\beta$-galactose. This site, close to the sialic acid-binding site, would favor the transfer reaction. Recent results suggest that the second alternative might be the correct one. First, kinetics of the sialidase and trans-sialidase reactions catalyzed by TcTS at different temperatures and with different donors do not support the formation of a more stable sialosyl-enzyme complex in the mechanism of transfer reaction, but suggest that the enzyme proceeds according to a sequential reaction mechanism (Ribeirao et al., 1997). Secondly, enzymatically inactive members of the trans-sialidase family, although unable to exhibit any sialidase and/or trans-sialidase activity, still bind to terminal $\beta$-galactose (Cremona et al., 1999).

The structural and mutagenesis results presented here now provide additional evidence suggesting the existence of distinct donor and acceptor binding sites (see Figure 5B) to account for the sialyltransferase activity of TcTS. An inhibitor-contacting aromatic residue (Tyr120) and a shallow depression (formed by Pro284 and Tyr249) are favorably positioned in TcTS to be involved in binding the acceptor molecule (Figure 4). Pro284 has previously been shown to be one of the essential amino acid residues for trans-sialylation, since a TrSA-TcTS chimeric molecule displaying only sialidase activity was able to transsialylate after mutation of Gln284 to a proline residue (Smith and Eichinger, 1997). In this work we tested both possibilities (Tyr120, Pro284) by site-directed mutagenesis. Our results indicate a crucial role for Tyr120 in binding the acceptor carbohydrate, since the single substitution of Tyr120 by Ser in TcTS dramatically changes the transfer/hydrolysis activity ratio towards a more efficient hydrolase, while the inverse substitution in $\operatorname{TrSA}$ retains a significant sialidase activity (Table III). Although these observations provide some indications about the overall location of galactose-binding sites in TcTS (Figure 5B), a more detailed description must await further crystallographic studies of enzyme-substrate complexes (to date, no such structure is available for microbial sialidases).

The use of enzymes in the synthesis of glycosidic linkages (Watt et al., 1998) has been shown to be an improvement over chemical methods, which remain long and low-yield processes. Although both glycosyltransferases and glycosidases can be used for this purpose, the latter have interesting advantages, such as their greater abundance and availability, and independence from expensive sugar-nucleotide donor substrates. However, glycosidases are in general unable to catalyze efficiently the transfer of sugar residues to acceptor molecules other than water. Extensive analyses of systems such as the sialidase/trans-sialidase model reported here will contribute to our understanding of the structural features capable of modulating a glycosidase scaffold towards an efficient transglycosidase activity.

Trans-sialidase has been studied extensively in T.cruzi (Frasch, 1994; Schenkman et al., 1994), the protozoan agent of Chagas disease in the American continent, but it has also been detected in Trypanosoma brucei, the agent of sleeping sickness in humans and nagana in domestic animals in Africa (Pontes de Carvalho et al., 1993a; Engstler et al., 1995), and in Endotrypanum species, a parasite of forest-dwelling tree sloths (Medina-Acosta et al., 1994). Given the involvement of trans-sialidase in T.cruzi infection of humans and the absence of a similar enzyme in eukaryotic cells, TcTS constitutes a good target for the development of compounds useful in controlling the infection. New alternatives for the chemotherapy of Chagas disease, which affects 16-18 million people in the Americas, are badly needed since the available compounds (nifurtimox and benznidazole) are only effective in $\sim 50 \%$ of acute infections and their usefulness for parasitological cure in chronic infections is controversial (Docampo and Schmunis, 1997). The structural changes responsible for the differences in sensitivity of TcTS to DANA might be exploited for the design of novel structure-based TcTS inhibitors. A similar approach in the case of influenza sialidases led to the design of potent antiviral inhibitors (von Itztein et al., 1993), which are now in phase III of clinical trials (Glaser, 1998).

\section{Conclusion}

The crystal structure of $\operatorname{TrSA}$ shows that trypanosomal sialidases and trans-sialidases fold into two structural 
domains: a canonical $\beta$-propeller fold where both the hydrolysis and transfer reactions take place; and a C-terminal lectin-like domain, which is probably involved in carbohydrate recognition. Structural and mutagenesis results allowed the identification of an aromatic residue in TcTS (Tyr120), which is crucial for binding the acceptor substrate. The presence of different binding sites for sialic acid donor and acceptor molecules in trans-sialidase may provide a mechanism to increase the efficiency of the transglycosylation reaction while still maintaining a double-displacement (ping-pong) reaction mechanism similar to that proposed for other neuraminidases (Chong et al., 1992).

\section{Materials and methods}

\section{Crystallization and data collection}

Natural TrSA was purified as described (Buschiazzo et al., 1997) except that MonoS was used instead of MonoQ in the FPLC purification step. The native enzyme $(7 \mathrm{mg} / \mathrm{ml})$ was crystallized by hanging drop vapor diffusion at $18^{\circ} \mathrm{C}$ with $17 \%$ PEG- $8000,100 \mathrm{mM}$ ammonium sulfate and $50 \mathrm{mM}$ morpholinoethanesulfonate $\mathrm{pH}$ 6.5. Crystals are orthorhombic, space group $P 22_{1} 2_{1} 2_{1}$, with cell dimensions $a=76.09 \AA, b=93.29 \AA$, $c=105.35 \AA$ and one molecule in the asymmetric unit. Crystals of the complex were obtained by soaking DANA into native crystals of TrSA. $\mathrm{X}$-ray data were collected from a single frozen crystal at $100 \mathrm{~K}$ in each case (Table I) using synchrotron radiation at EMBL-DESY (Hamburg, Germany) and LURE (Paris, France), and reduced using the programs DENZO and SCALEPACK (Otwinowski and Minor, 1997).

\section{Structure determination and refinement}

The structure was determined by a combination of molecular replacement methods with the program AMoRe (Navaza, 1994) using the structure of S.typhimurium sialidase [Protein Data Bank (PDB) code 2SIM; Crennell et al., 1993] as search probe, and heavy atom methods using a single mercurial derivative $(0.5 \mathrm{mM} \mathrm{HgCl}$, overnight soaking). Data for the $\mathrm{Hg}$ derivative were measured at $3.0 \AA$ A resolution using a rotating anode X-ray generator (141 689 measured reflections, 15581 independent reflections, $99.2 \%$ complete, $R$-merge $=13.4 \%$ ). All further calculations were carried out with programs from the CCP4 package (Collaborative Computational Project Number 4, 1994), unless otherwise indicated. Heavy atom binding sites were associated with the three free cysteine residues of TrSA (positions 229, 286 and 439) and were refined with the program MLPHARE. A solvent-flattened map calculated with SIR phases allowed polypeptide chain tracing for most of the catalytic domain, except for the outermost $\beta$-strands and several connecting loops. This map also revealed a $\beta$-sandwich structure for the $\mathrm{C}$-terminal domain, but only the central regions of the $\beta$-strands were modeled at this stage. Subsequent cycles of refinement with the program REFMAC and modeling with the program O (Jones et al., 1991) using maps calculated with combined (model + SIR) phases allowed complete polypeptide chain tracing and introduction of well-defined water molecules. The free $R$-factor (Brünger, 1992) was used throughout to monitor progress of the refinement. The final protein model (Table I) includes 622 amino acid residues (residues 404-409 and 591-593 in two exposed loops, and 632-638 at the C-terminus are not visible in density) and five GlcNAc residues covalently attached to the protein. The stereochemical quality of the model is comparable to that of other structures refined at a similar resolution: 526 out of 534 non-glycine residues (98.5\%) display mainchain dihedral angles that fall within the most favored or additionally allowed regions of the Ramachandran plot as defined by the program PROCHECK.

The bound inhibitor was located from a Fourier difference map calculated with model phases. The structure of the TrSA-DANA complex was refined as described above for unliganded $\operatorname{TrSA}$, and the parameters after the final refinement cycle are shown in Table I. Atomic coordinates and structure factor data have been deposited with the PDB and are available from the authors on request.

\section{Homology modeling of T.cruzi trans-sialidase}

TcTS was modeled by maintaining the protein backbone conformation of TrSA, since the two proteins have $70 \%$ amino acid identity and there are no insertions or deletions except from a single residue, Ser23, in a solvent-exposed loop of $\operatorname{TrSA}$ that is absent in TcTS. Amino acid substitutions were introduced into the TrSA framework using the rotamer library implemented in program $\mathrm{O}$, a few close contacts were corrected manually, and the entire model was subjected to energy minimization using the CHARMM force field as implemented in Quanta (MSI).

\section{Site-directed mutagenesis}

Site-directed point mutagenesis was performed using the Chameleon Double-Stranded site-directed mutagenesis kit (Stratagene, La Jolla, CA), according to the manufacturer's instructions. Mutagenic oligonucleotides were synthesized minimizing the number of base changes in order to achieve the codon modification. Double-stranded recombinant pTrcHisA (Invitrogen, San Diego, CA) plasmids were used as templates in the mutagenesis reactions: clone pTrcTS611/2 (Buschiazzo et al., 1997) containing an insert encoding the globular non-repetitive core of T.cruzi trans-sialidase; and clone pTrcTrSA4_Nhe [which derives from clone pTrSA4 (Buschiazzo et al., 1997), by PCR-subcloning into restriction site NheI of plasmid pTrcHisA] containing an insert encoding the sialidase of T.rangeli. Both clones encode fusion recombinant proteins containing the vector-encoded His tag at the N-terminus.

Recombinant protein expression and enzyme activity assays The mutagenized clones were used to transform Escherichia coli XL 1 blue. After growth in LB broth, with constant agitation at 250 r.p.m. at $37^{\circ} \mathrm{C}$, up to $\mathrm{Abs}_{600} 0.6-0.8$, bacteria were induced to overexpress recombinant protein by adding $0.5 \mathrm{mM}$ isopropyl- $\beta$-D-thiogalactopyranoside (Sigma, St Louis, MO). Induction was maintained with normal agitation at $28^{\circ} \mathrm{C}$ for $12-16 \mathrm{~h}$. Cells were harvested and frozen $\left(-80^{\circ} \mathrm{C}\right)$ until needed. After thawing, lysis was achieved in the presence of $1 \mathrm{mM}$ phenylmethylsulfonyl fluoride and $5 \mathrm{mM}$ EDTA, using a Sonifier 450 sonicator (Branson, Danbury, CT), giving five 15 -s pulses at $60 \%$ maximal power. Supernatants were centrifuged at $21000 \mathrm{~g}$ for $30 \mathrm{~min}$ and subjected to iminodiacetic acid metal affinity chromatography (IMAC) $\mathrm{Ni}^{2+}$-charged. Elution was achieved using $100 \mathrm{mM}$ imidazole in $20 \mathrm{mM}$ Tris- $\mathrm{HCl} \mathrm{pH} 8.0,0.5 \mathrm{M} \mathrm{NaCl}$. Proteins were analyzed by SDS-PAGE under reducing conditions, stained with Coomassie Blue R250 and quantitated with Image Quantifier 3.22 (Molecular Dynamics) using purified bovine serum albumin as standard. Protein expression yield was similar for all mutants as judged by SDS-PAGE (not shown).

Enzyme activity assays were carried out using the purified proteins as described previously (Buschiazzo et al., 1997). Briefly, trans-sialidase was measured in $20 \mathrm{mM}$ Tris- $\mathrm{HCl} \mathrm{pH} 7.6,30 \mathrm{mM} \mathrm{NaCl}, 1 \mathrm{mM}$ 3'-sialyllactose and $12 \mu \mathrm{M}\left[{ }^{14} \mathrm{C}\right]$ lactose $(55 \mathrm{mCi} / \mathrm{mmol})$, in $30 \mu \mathrm{l}$ final volume at $25^{\circ} \mathrm{C}$. The reaction was stopped, and sialyl- $\left[{ }^{14} \mathrm{C}\right]$ lactose was quantitated with a $\beta$-scintillation counter. Sialidase was measured in $20 \mathrm{mM}$ Tris- $\mathrm{HCl} \mathrm{pH} \mathrm{7.6,} 30 \mathrm{mM} \mathrm{NaCl}, 0.2 \mathrm{mM}$ 4-methylumbelliferyl- $N$ acetylneuraminic acid, in $50 \mu \mathrm{l}$ final volume at $25^{\circ} \mathrm{C}$. The reaction was stopped, and fluorescence of free 4-methylumbelliferone was measured in $0.2 \mathrm{M}$ carbonate $\mathrm{pH} 10$ with a DYNA Quant ${ }^{\mathrm{TM}} 200$ Fluorometer (Hoefer Pharmacia Biotech, Uppsala, Sweden).

\section{Acknowledgements}

We thank G.A.Bentley, F.Saul, A.Parodi and J.J.Cazzulo for critical comments on the manuscript. This work was supported by grants from the World Bank/UNDP/WHO Special Program for Research and Training in Tropical Diseases (TDR), ECOS-SeCyT (France-Argentina), the Institut Pasteur (France), the Consejo Nacional de Investigaciones Científicas y Técnicas and the Agencia Nacional de Promoción Científica y Tecnológica (Argentina). We thank the European Union for support under the TMR/LSF program to the EMBL Hamburg Outstation, reference number ERBFMGECT980134. The research by A.C.C.F. was supported in part by an International Research Scholars Grant from the Howard Hughes Medical Institute.

\section{References}

Brünger,A.T. (1992) Free $R$ value: a novel statistical quantity for assessing the accuracy of crystal structures. Nature, 355, 472-474.

Burmeister,W.T., Henrissat,B., Bosso,C., Cusack,S. and Ruigrok,R.W.H. (1993) Influenza B virus neuraminidase can synthesize its own inhibitor. Structure, 1, 19-26.

Buschiazzo,A., Cremona,M.L., Campetella,O.D., Frasch,A.C.C. and Sánchez,D.O. (1993) Sequence of a Trypanosoma rangeli gene closely related to Trypanosoma cruzi trans-sialidase. Mol. Biochem. Parasitol., 62, 115-116. 
Buschiazzo,A., Campetella,O. and Frasch,A.C.C. (1997) Trypanosoma rangeli sialidase: cloning, expression and similarity to T. cruzi transsialidase. Glycobiology, 7, 1167-1173.

Campetella,O., Uttaro,A., Parodi,A.J. and Frasch,A.C.C. (1994) A recombinant Trypanosoma cruzi trans-sialidase lacking the amino acid repeats retains the enzymatic activity. Mol. Biochem. Parasitol., 64, 337-340.

Chong,A.K.J., Pegg,M.S., Taylor,N.R. and von Itzstein,M. (1992) Evidence for a sialosyl cation transition-state complex in the reaction of sialidase from influenza virus. Eur. J. Biochem., 207, 335-343.

Chuenkova,M., Pereira,M.E. and Taylor,G. (1999) Trans-sialidase of Trypanosoma cruzi: location of galactose-binding site(s). Biochem. Biophys. Res. Commun., 262, 549-556.

Collaborative Computational Project Number 4 (1994) The CCP4 suite: programs for protein crystallography. Acta Crystallogr. D, 50, 760-763.

Cremona,M.L., Sánchez,D.O., Frasch,A.C.C. and Campetella,O. (1995) A single tyrosine differentiates active and inactive Trypanosoma cruzi trans-sialidases. Gene, 160, 123-128.

Cremona,M.L., Campetella,O., Sánchez,D.O. and Frasch,A.C.C. (1999) Enzymically inactive members of the trans-sialidase family from Trypanosoma cruzi display $\beta$-galactose binding activity. Glycobiology, 9, 581-587.

Crennell,S.J., Garman,E.F., Graeme Laver,W., Vimr,E.R. and Taylor,G.A. (1993) Crystal structure of a bacterial sialidase (from Salmonella typhimurium LT2) shows the same fold as an influenza virus neuraminidase. Proc. Natl Acad. Sci. USA, 90, 9852-9856.

Crennell,S.J., Garman,E., Graeme Laver,W., Vimr,E. and Taylor,G. (1994) Crystal structure of Vibrio cholerae neuraminidase reveals dual lectin-like domains in addition to the catalytic domain. Structure, $\mathbf{2}$, 535-544.

Crennell,S.J., Garman,E.F., Philippon,C., Vasella,A., Graeme Laver,W., Vimr,E.R. and Taylor,G.A. (1996) The structures of Salmonella typhimurium LT2 neuraminidase and its complexes with three inhibitors at high resolution. J. Mol. Biol., 259, 264-280.

D'Alessandro-Bacigalupo,A. and Gore Saravia,N. (1992) Trypanosoma rangeli. In Kreier,J.P. and Baker,J.R. (eds), Parasitic Protozoa. Academic Press, San Diego, CA, Vol. 2, pp. 1-54.

Docampo,R. and Schmunis,G.A. (1997) Sterol biosynthesis inhibitors: potential chemotherapeutics against Chagas disease. Parasitol. Today, 13, 129-130.

Engstler,M., Schauer,R. and Brun,R. (1995) Distribution of developmentally regulated trans-sialidases in the Kinetoplastida and characterization of a shed trans-sialidase activity from procyclic Trypanosoma congolense. Acta Trop., 59, 117-129.

Ferrero-García,M.A., Trombetta,S.E., Sánchez,D.O., Reglero,A., Frasch,A.C.C. and Parodi,A.J. (1993) The action of Trypanosoma cruzi trans-sialidase on glycolipids and glycoproteins. Eur. J. Biochem., 213, 765-771.

Frasch,A.C.C. (1994) Trans-sialidase, SAPA amino acid repeats and the relationship between Trypanosoma cruzi and the mammalian host. Parasitology, 108, S37-44.

Glaser,V. (1998) Neuraminidase inhibitors take bite out of influenza. Nature Biotechnol., 16, 1002.

Henrissat,B. (1998) Glycosidase families. Biochem. Soc. Trans., 26, $153-156$.

Henrissat,B. and Bairoch,A. (1993) New families in the classification of glycosyl hydrolases based on amino acid sequence similarities. Biochem. J., 293, 781-788.

Holm,L. and Sander,C. (1994) Searching protein structure databases has come of age. Proteins, 19, 165-173.

Hoyer,L.L., Hamilton,A.C., Steenbergen,S.M. and Vimr,E.R. (1992) Cloning, sequencing and distribution of the Salmonella typhimurium LT2 sialidase gene, nanH, provides evidence for interspecies gene transfer. Mol. Microbiol., 6, 873-884.

Jones,T.A., Zou,J.Y., Cowan,S.W. and Kjeldgaard,M. (1991) Improved methods for binding protein models in electron density maps and the location of errors in these models. Acta Crystallogr. A, 47, 110-119.

Koshland,D.E. (1953) Stereochemistry and the mechanism of enzymatic reactions. Biol. Rev. 28, 416-436.

Laskowski,R.A., MacArthur,M.W., Moss,D.S. and Thornton,J.M. (1993) PROCHECK: a program to check the stereochemical quality of protein structures. J. Appl. Crystallogr., 26, 283-291.

Luo,Y., Li,S.C., Chou,M.Y., Li,Y.T. and Luo,M. (1998) The crystal structure of an intramolecular trans-sialidase with a NeuAc $\alpha 2 \rightarrow 3 \mathrm{Gal}$ specificity. Structure, 15, 521-530.

Medina-Acosta,E., Franco,A.M., Jansen,A.M., Sampol,M., Neves,N. Pontes de Carvalho,L., Grimaldi Junior,G. and Nussenzweig,V. (1994)
Trans-sialidase and sialidase activities discriminate between morphologically indistinguishable trypanosomatids. Eur. J. Biochem., $\mathbf{2 2 5}, 333-339$.

Navaza,J. (1994) AMoRe: an automated package for molecular replacement. Acta Crystallogr. A, 50, 157-163.

Otwinowski,Z. and Minor,W. (1997) Processing of X-ray diffraction data collected in oscillation mode. Methods Enzymol., 276, 307-325.

Parodi,A.J., Pollevick,G.D., Mautner,M., Buschiazzo,A., Sánchez,D.O. and Frasch,A.C.C. (1992) Identification of the gene(s) coding for the trans-sialidase of Trypanosoma cruzi. EMBO J., 11, 1705-1710.

Paulson,J.C. and Colley,K.J. (1989) Glycosyltranferases. Structure, localization and control of cell type-specific glycosylation. J. Biol. Chem., 264, 17615-17618.

Pereira,M.E.A., Mejia,S., Ortega-Barria,E., Matzilevich,D. and Prioli,R.P. (1991) The Trypanosoma cruzi neuraminidase contains sequences similar to bacterial neuraminidases, YWTD repeats of the low density lipoprotein receptor and type III modules of fibronectin. J. Exp. Med., 174, 179-191.

Pollevick,G.D., Affranchino,J.L., Frasch,A.C.C. and Sánchez,D.O. (1991) The complete sequence of SAPA, a shed acute-phase antigen of Trypanosoma cruzi. Mol. Biochem. Parasitol., 47, 247-250.

Pontes de Carvalho,L.C., Tomlinson,S., Vandekerckhove,F., Jay Bienen,E., Clarkson,A.B., Jiang,M.S., Hart,G.W. and Nussenzweig,V. (1993a) Characterization of a novel trans-sialidase of Trypanosoma brucei procyclic trypomastigotes and identification of procyclin as the main sialic acid acceptor. J. Exp. Med., 177, 465-474.

Pontes de Carvalho,L.C., Tomlinson,S. and Nussenzweig,V. (1993b) Trypanosoma rangeli sialidase lacks trans-sialidase activity. Mol. Biochem. Parasitol., 62, 19-26.

Previato,J.O., Andrade,A.F., Pessolani,M.C. and Mendonca Previato,L. (1985) Incorporation of sialic acid into Trypanosoma cruzi macromolecules. A proposal for a new metabolic route. Mol. Biochem. Parasitol., 16, 85-96.

Ribeirao,M., Pereira-Chioccola,V.L., Eichinger,D., Rodrigues,M.M. and Schenkman,S. (1997) Temperature differences for trans-glycosylation and hydrolysis reactions reveal an acceptor binding site in the catalytic mechanism of Trypanosoma cruzi trans-sialidase. Glycobiology, $\mathbf{7}$, 1237-1246

Roggentin,P., Schauer,R., Hoyer,L.L. and Vimr,E.R. (1993) The sialidase superfamily and its spread by horizontal gene transfer. Mol. Microbiol., 9, 915-921.

Schenkman,S., Man-Shiow,J., Hart,G.H. and Nussenzweig,V. (1991) A novel cell surface trans-sialidase of Trypanosoma cruzi generates a stage-specific epitope required for invasion in mammalian cells. Cell, 65, 1117-1125.

Schenkman,S., Eichinger,D., Pereira,M.E.A. and Nussenzweig,V. (1994) Structural and functional properties of Trypanosoma trans-sialidase. Annu. Rev Microbiol, 48, 499-523.

Smith,L.E. and Eichinger,D. (1997) Directed mutagenesis of the Trypanosoma cruzi trans-sialidase enzyme identifies two domains involved in its sialyltransferase activity. Glycobiology, 7, 445-451.

Smith,L.E., Uemura,H. and Eichinger,D. (1996) Isolation and expression of an open reading frame encoding sialidase from Trypanosoma rangeli. Mol. Biochem. Parasitol., 79, 21-33.

Taylor,G. (1996) Sialidases: structures, biological significance and therapeutic potential. Curr. Opin. Struct. Biol., 6, 830-837.

Tomlinson,S. and Raper,J. (1998) Natural immunity to trypanosomes. Parasitol. Today, 14, 354-359.

Uemura,H., Schenkman,S., Nussensweig,V. and Eichinger,D. (1992) Only some members of a gene family in Trypanosoma cruzi encode proteins that express both trans-sialidase and neuraminidase activities. EMBO J., 11, 3837-3844.

Vandekerckhove,F., Schenkman,S., Pontes de Carvalho,L., Tomlinson,S., Kiso,M., Yoshida,M., Hasegawa,A. and Nussenzweig,V. (1992) Substrate specificity of the Trypanosoma cruzi trans-sialidase. Glycobiology, 2, 541-548.

Varghese,J.N. Laver,W.G. and Colman,P.M. (1983) Structure of the influenza virus glycoprotein antigen neuraminidase at $2.9 \AA$ resolution. Nature, 303, 35-40.

Vimr,E.R. (1994) Microbial sialidases: does bigger always mean better? Trends Microbiol., 2, 271-277.

von Itztein,M. et al. (1993) Rational design of potent sialidase-based inhibitors of influenza virus replication. Nature, 363, 418-423.

Watt,G.M., Lowden,P.A.S. and Flitsch,S.L. (1998) Enzyme-catalyzed formation of glycosidic linkages. Curr. Opin. Struct. Biol., 7, 652-660.

Received August 27, 1999; revised November 2, 1999; accepted November 3, 1999 